\title{
Gray-Scale Liver Enhancement with Sonazoid (NC100100), a Novel Ultrasound Contrast Agent; Detection of Hepatic Tumors in a Rabbit Model
}

\author{
Rira Watanabe, ${ }^{*}$ Manabu Matsumura, Chun-Jen Chen, Yuki Kaneda, Masanao Ishihara, and \\ Masayoshi FUJIMAKI
}

Daiichi Pharmaceutical Co., Ltd.; 1-16-13 Kita-kasai, Edogawa-ku, 134-8630 Tokyo, Japan. Received February 5, 2003; accepted June 20, 2003; published online June 27, 2003

\begin{abstract}
The liver contrast effects of Sonazoid in two ultrasonographic imaging modes, gray-scale conventional and harmonic, were examined as a time-related study in normal rabbits, and evaluated quantitatively and visually with tumor-model rabbits to estimate the diagnostic potential. Peak enhancement of vessels and parenchyma was observed $1 \mathrm{~min}$ after injection in both modes, although signal enhancement in the parenchyma lasted for $120 \mathrm{~min}$ compared with rapid decay (5-10 $\mathrm{min})$ in vessels. When Sonazoid was intravenously injected into metastatic carcinoma-model (VX-2) rabbits, all hepatic tumors showed ring enhancement in the early phase followed by clear contrast defects in the delayed phase, because signal enhancement remained only in normal parenchyma. Visual analysis scores for the diagnosis of tumors were improved by Sonazoid injection, and the videodensitometric differences between tumor and normal tissues were significantly greater after injection. Although the harmonic mode tended to show better contrast effects, the conventional mode provided significant contrast enhancement in this hepatic tumor-model. Sonazoid might be useful for the detection of undifferentiated tumors in the liver by making it possible to visualize neovascularity in the early phase and clear contrast defects in the delayed phase, not only in the harmonic but also in the conventional mode.
\end{abstract}

Key words ultrasound contrast agent; Sonazoid; NC100100; liver; VX-2; rabbit

Ultrasonography (US) is useful for the diagnosis of hepatic focal lesions on the basis of their distinctive echogenicities, the gray-scale morphologic features. Some kinds of malignant tumors, however, especially small ones, have a low echogenitic difference in comparison with the surrounding healthy tissue (so-called 'isoechoic'), so that the detection of these tumors is not always possible. Detection and characterization of tumor vascularity are important for the differential diagnosis of a liver mass. ${ }^{1-3)}$ Although the color or power Doppler mode helps in diagnosis by visualizing blood flows, ${ }^{4}$-7) it does not enable dynamic study or visualization of fine vessels as clearly as do dynamic computed tomography or magnetic resonance imaging (MRI) ${ }^{1)}$ In recent years, as US contrast agents and novel technologies for ultrasonic imaging have been developed, US with some microbubblebased contrast agents has rendered possible the visualization of tissue perfusion in gray-scale harmonic imaging, a novel technique based on the nonlinear backscatter property of microbubbles. $^{8-10)}$

A new US contrast agent Sonazoid (NC100100), comprising a suspension of microbubbles of a low solubility inert gas, ${ }^{11)}$ has been reported to enhance tissue perfusion, especially in the myocardium, ${ }^{12)}$ by conventional gray-scale imaging as well as by harmonic imaging. In addition to showing high echogenicity, this agent is said to be well incorporated by reticuloendothelial systems (RES) and enhances the liver parenchymal imaging in the delayed phase. ${ }^{13)}$ This "parenchyma-specific imaging" in the delayed phase is suggested to improve the detection and delineation of focal liver tumors, because malignant tumors have few or no RES and will show-up as defects. ${ }^{14)}$

For this study, the liver contrast effects of this agent were evaluated by quantitative measurement of videodensity and visual analysis in gray-scale conventional and harmonic imaging modes. Videodensity was initially measured in normal rabbits to determine the timing for delayed phase evaluation. Secondly, the gray-scale contrast between tumor and normal tissue measured in parallel with tumor detection and delineation was evaluated by visual analysis in VX-2-implanted rabbits, ${ }^{15,16)}$ a hepatic tumor model. ${ }^{17)}$

\section{MATERIALS AND METHODS}

Ultrasound Contrast Agent Sonazoid (Amersham Health, Oslo, Norway) consists of perfluorobutane microbubbles stabilized with a lipid shell. ${ }^{11)}$ The microbubbles have a relatively narrow size-distribution, with a median diameter of around 2 to $3 \mu \mathrm{m}$, and the concentration is approximately $1 \times 10^{9}$ particles $/ \mathrm{ml}$. In a preliminary liver imaging study in rabbits, Sonazoid showed definite parenchymal enhancement in doses ranging from 0.045 to $0.315 \mu \mathrm{l}$ microbubbles $/ \mathrm{kg}$; and there was no significant difference within this range. Over-doses inhibit ultrasound penetration in deeper zones of the liver and cause contrast attenuation, so that $0.1 \mu \mathrm{l}$ mirobubbles $/ \mathrm{kg}$ was considered suitable as an anticipated clinical dose and was selected throughout these studies.

Animal and Instrumental Preparation All experimental procedures were performed in accordance with the inhouse guidelines of the Institutional Animal Care and Use Committee of Daiichi Pharmaceutical Co., Ltd. Male New Zealand White rabbits (Oriental Yeast, Tokyo, Japan) with body weights of 2.5 to $3.0 \mathrm{~kg}$ were used for the imaging study, and a female Japanese White rabbit (Funabashi Farm, Funabashi, Japan) bearing VX-2 in its thigh was used as the donor. US of the livers and preparation of a hepatic tumor model were conducted under anesthesia by intravenous injection of pentobarbital sodium $(20 \mathrm{mg} / \mathrm{kg})$ and inhalation of halothane. 
We performed US by an ultrasound scanner (PV 7000; Toshiba, Tokyo, Japan) equipped with an electric convex probe. The transmit/receive center frequencies were 3.0/3.0 megahertz $(\mathrm{MHz})$ in the conventional mode and $2.1 / 4.5 \mathrm{MHz}$ in the harmonic mode with acoustic power of 0.2 Mechanical Index (MI) and 0.4 MI, respectively. The frame rate was designated as 19 to 21 frames per second (fps) in continuous imaging and $0.4 \mathrm{fps}$ in intermittent imaging in both modes. The other parameters, such as gray-scale gain, were recorded and maintained throughout each injection. Ultrasound images were recorded continuously on S-VHS videotapes for off-line analysis. Rabbits were shaved on the upper abdomen and were placed in a supine position for percutaneous US. An injection of contrast agent was given though a 22-gauge catheter cannulated in an ear vein. The tubing was flushed with $3 \mathrm{ml}$ of saline just after the contrast agent injection.

Time Response Study Six of 12 rabbits were used for the conventional mode and the others for the harmonic mode.

The liver was first scanned with continuous imaging at pre-injection (baseline) and consecutively initial post-injection: 0 to $30 \mathrm{~s}$ after injection of Sonazoid. The change of contrast effect with time was assessed with intermittent imaging in order to minimize destruction of the microbubbles by ultrasound from $30 \mathrm{~s}$ to $1 \mathrm{~min}$ after injection and the last three frames were used for quantitative analysis. Subsequently, the first three frames were acquired with intermittent imaging at $3,5,10,20,30,60,120 \mathrm{~min}$ and $24 \mathrm{~h}$ after injection.

VX-2 Detectability Study VX-2 tumor tissue cubes of approximately $2 \mathrm{~mm}^{3}$ were obtained from the donor rabbit and implanted into the livers of sixteen rabbits. As three animals died before the imaging study was performed, from 15 to $19 \mathrm{~d}$ after implantation, thirteen were used: six for the conventional mode and the other seven for the harmonic mode.

Before the injection of Sonazoid, the liver was first assessed by continuous imaging in the conventional mode, because lesion discrimination was difficult in the harmonic mode. After the imaging plane was fixed to include the tumor, the tumors were imaged by continuous imaging until $30 \mathrm{~s}$ after the injection of Sonazoid, in order to investigate the continuous change of contrast effects in detail, and subsequently with intermittent imaging up to 1 min after injection in both modes (early phase). At $10 \mathrm{~min}$ after injection, the tumors were imaged by the same intermittent imaging for three frames for quantitative analysis and subsequent continuous imaging in both modes (delayed phase).

After the imaging study, the animals were killed by injection of a lethal dose of pentobarbital sodium. Autopsies were performed to confirm the presence of tumors in the liver lobes and to provide anatomic correlation with the US observations.

Analysis and Statistics For quantitative analysis, US images on the videotapes were acquired by a computer programmed with a video frame grabber. An off-line image analysis system (Color Cardiology Work Station; Freeland systems, Boulder, CO, U.S.A.) was used for calculating videodensity. In this system, the gray-scale level ranged from 0 to 255 . Videodensities were taken of the appropriate regions of interest (ROIs), which included the normal liver parenchyma, vessels and the tumor, and respective evaluations were made. All videodensity data were expressed as the mean \pm S.D. Statistical analysis was performed by using one- way analysis of variance and Dunnett's multiple comparison tests. The results were considered significant at $p<0.05$.

In the VX-2 detectability study, visual assessment was also performed. Each lesion was assessed before injection (conventional mode) and during the early and delayed phases by three independent observers, retrospectively, by using videotape playback. Contrast images for a total of 18 or 21 (6 or 7 rabbits $\times 3$ observers) observations were obtained in the conventional or harmonic mode.

The liver lesions detected during imaging were subjectively classified according to the following criteria ${ }^{18)}$ :

Type A: a clear difference in echogenicity between lesion and normal tissue with presence of a tumor.

Type B: a small difference in echogenicity between lesion and normal tissue with high suspicion of tumor presence.

Type C: the lesion is isoechoic with the tissue; however, it can be recognized as a mass moving differentially from the surrounding normal tissue.

Type D: no lesion is detected, or detection is impossible due to lack of echoic homogeneity of the normal tissue and/or artifacts.

In the same way, delineation of the lesions was subjectively assessed by the following scoring system: 3 , excellent with complete delineation of the lesion; 2, acceptable but part of the lesion not well delineated; 1, poor, insufficient delineation.

\section{RESULTS}

Time Response Study In both conventional and harmonic modes, the liver vasculature and parenchyma were clearly enhanced in all rabbits after the administration of Sonazoid. Visually, the vessels were first enhanced, and then within a few seconds the parenchyma was subsequently enhanced. Peak enhancement of vessels and parenchyma was observed 1 min after injection in both modes (Figs. 1, 2). As signal enhancement in vessels decayed rapidly, the videodensity in the vessels was statistically higher than baseline only at 1 min (conventional mode) or, at 1 and 3 min after injection (harmonic mode). Conversely, videodensity in the parenchyma decreased slowly and lasted for more than 120 min in both modes. No signal enhancement was recognized $24 \mathrm{~h}$ after injection of the contrast agent.

VX-2 Detectability Study VX-2 model rabbits had liver tumors ranging in size from 4 to $18 \mathrm{~mm}$ at the time of the imaging study. The VX-2 tumors could be recognized as a low echoic mass; however, detection and delineation were difficult because the lesion was almost isoechoic with the normal tissue and boundaries of the masses were unclear before the injection of Sonazoid (Figs. 3a, 4a). Shortly after injection of the agent, the main vessels and tumor rim were enhanced (Figs. 3b, 4b), and then the tumors were wholly enhanced except for the central portions of some tumors. Subsequently, the entire liver was enhanced, but the tumor enhancement decayed rapidly even within 1 min after the contrast injection. Although the enhancement of vessels and tumors had already disappeared at $10 \mathrm{~min}$ after the contrast injection, the echogenicity of the normal tissue remained high. Therefore, the liver lesions continued to show clear contrast defects in the delayed phase (Figs. 3c, 4c). On histological examination, the central portions of some VX-2 tumors were 


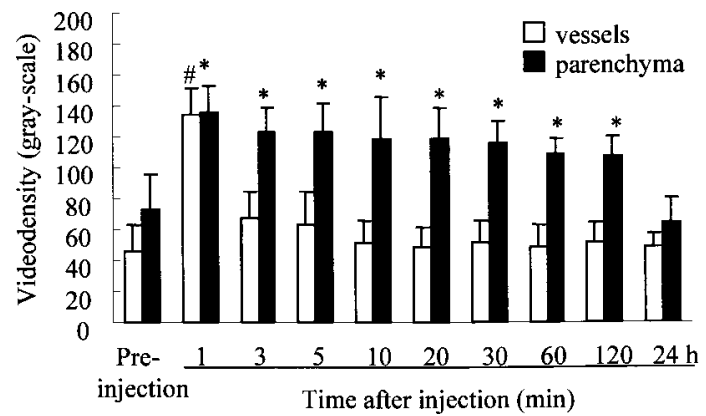

Fig. 1. Videodensitometric Measurements in the Vessels (Open) and Parenchyma (Closed) of the Liver after Injection of Sonazoid in Rabbits $(0.1 \mu 1$ Microbubbles $/ \mathrm{kg}$, Conventional Mode)

Differences when $* p<0.05$ (parenchyma) and $\# p<0.05$ (vessel) were $v s$. the respective pre-injection values (Dunnett)

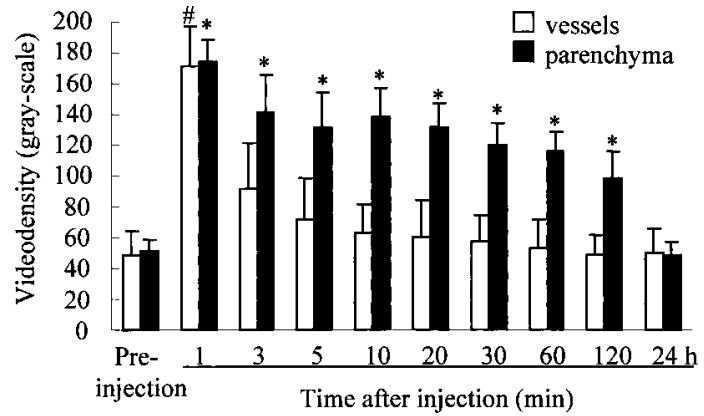

Fig. 2. Videodensitometric Measurements in the Vessels (Open) and Parenchyma (Closed) of the Liver after Injection of Sonazoid in Rabbits (0.1 $\mu$ l Microbubbles $/ \mathrm{kg}$, Harmonic Mode)

Differences when $* p<0.05$ (parenchyma) and ${ }^{\#} p<0.05$ (vessel) were $v s$. the respective pre-injection values (Dunnett).

necrotic, and only the peripheral portions were viable (Figs. $3 \mathrm{~d}, 4 \mathrm{~d})$. While the necrotic lesions were consistent with the non-enhanced areas in the early and delayed phases, the viable lesions were enhanced in the early phase. Whole lesions of the tumors corresponded well with the contrast defects in the delayed phase.

The videodensity of normal liver parenchyma increased after the injection in both modes, whereas that of the tumor did not change (Figs. 5, 6). The values of normal tissues were significantly higher in both early and delayed phases compared with the baseline, and no difference between early and delayed phases was seen. The quantitative contrast in the videodensitometric measurements between the tumor and normal tissue was significantly higher after injection compared with the baseline in both modes, and that value was also higher in the harmonic than in the conventional mode (Fig. 7). However, no statistical difference was detected between the early and delayed phases.

Independent observers judged that detection and delineation of the lesion were improved after the injection of Sonazoid (Table 1). Concerning the detection, positively identified tumors (Type A) increased from 0 (baseline) to 2 (early phase) and 7 (delayed phase) of 18 observations in the conventional mode, and from 0 (baseline) to 7 (early phase) and 12 (delayed phase) of 21 observations in the harmonic mode. With respect to delineation, excellent and complete delineation of the tumor (score 3 ) increased from 0 (baseline) to 1 (early phase) and 7 (delayed phase) of 18 observations in the conventional mode, and from 1 (baseline, conventional mode) to 6 (early phase) and 12 (delayed phase) of 21 observations in the harmonic mode. The judgment tended to be more discriminative in the harmonic than the conventional mode, and better in the delayed than the early phase.

\section{DISCUSSION}

Sonazoid is a novel microbubble-based ultrasound contrast agent that strongly enhances echogenicity for echocardiography and other types of sonography. ${ }^{12,19-21)}$ It is classified as a second-generation agent ${ }^{22}$ in which the perfluorocarbon gas has a solubility considerably lower than air, resulting in extended stability in vivo. Sonazioid has been known to have unique "delayed imaging" in addition to "vascular imaging" properties in vivo; it provides contrast enhancement in vessels and tissue perfusion immediately after injection as with other blood-pool type contrast agents, and then high echogenic contrast enhancement in the parenchyma as it gradually disappears from the blood circulation. Since Sonazoid microbubbles are thought to be taken up by the RES, especially via phagocytosis by Kupffer cells, this "delayed imaging" is expected to be useful for detecting malignant tumors, which lack Kupffer cells.

We first examined the duration of contrast enhancement in vessels and liver parenchyma of normal rabbits. Videodensitometric measurements (Figs. 1, 2) showed that the parenchyma maintained high echogenicity up to $120 \mathrm{~min}$, while the signal in vessels decreased rapidly. Because it is generally necessary to visually recognize changes in signal of more than 20 units in the 256 gray-scales, ${ }^{23)} 5$ (conventional mode) or 10 (harmonic mode) to 120 min after injection was considered appropriate timing for the "delayed imaging" in this study. Although further investigations might be required to optimize timing of the delayed phase for other conditions, such as species, equipment, and ultrasonographic technique, the time window for the delayed phase of this agent is quite long enough to permit scanning of the whole liver precisely without any practical time limitation.

Perfluorocarbon formulated in microbubbles, or in emulsion, is known to be eliminated through exhalation without being metabolized when it is administered intravenously; it dissolves slowly into blood and is subsequently exhaled through the membrane of alveolus. ${ }^{24)}$ Therefore, the disappearance of parenchymal contrast $24 \mathrm{~h}$ after injection is considered a result of dissolution of gas and simultaneous shrinkage of the microbubbles staying in the liver parenchyma. As it has been postulated so far that the parenchymal contrast might be due to the accumulation of the microbubbles in Kupffer cells in the liver, a faster disappearance of vessel enhancement suggested that the microbubbles might be more stable in the cells than in the blood stream.

In the second examination in which VX-2 model rabbits were used, we chose $10 \mathrm{~min}$ after injection for "delayed imaging." While videodensitometric measurements (Figs. 5, 6) showed similar contrast enhancement in both early and delayed phases, the scores for both detection and delineation tended to be higher in the latter by visual judgment (Table 1). This discrepancy might be due to strong signals in vessels around the tumor, disturbing the visual distinction between 
a)

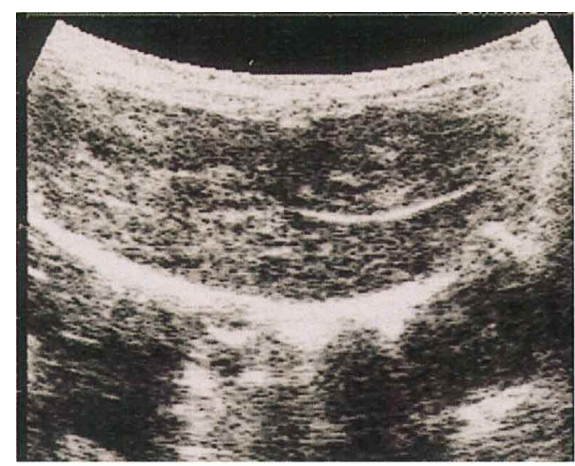

c)

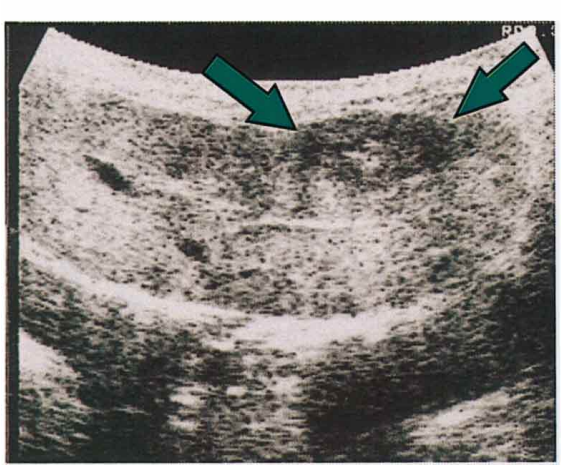

b)

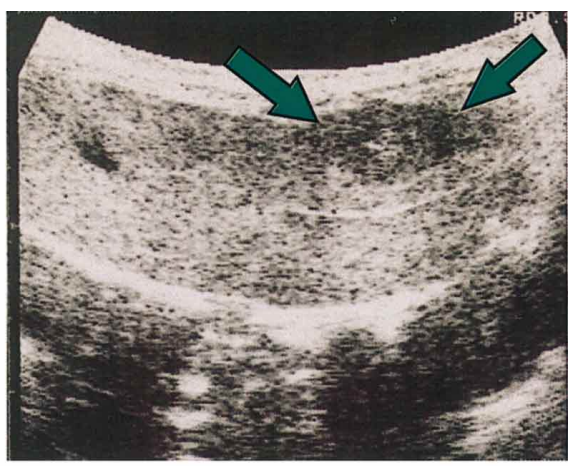

d)

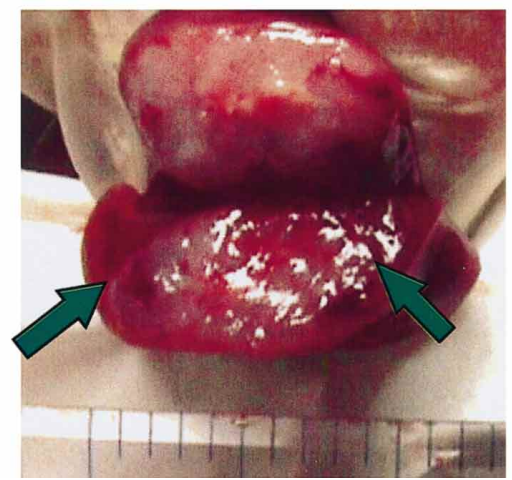

Fig. 3. The Liver of a VX-2 Tumor-Bearing Rabbit Imaged in the Conventional Mode before (a), Immediately after (b), and $10 \mathrm{~min}$ after (c) Injection of $0.1 \mu 1$ Microbubbles $/ \mathrm{kg}$ of Sonazoid

Arrows show the VX-2 tumor. The tumor was identified by macroscopic observation (d).

a)

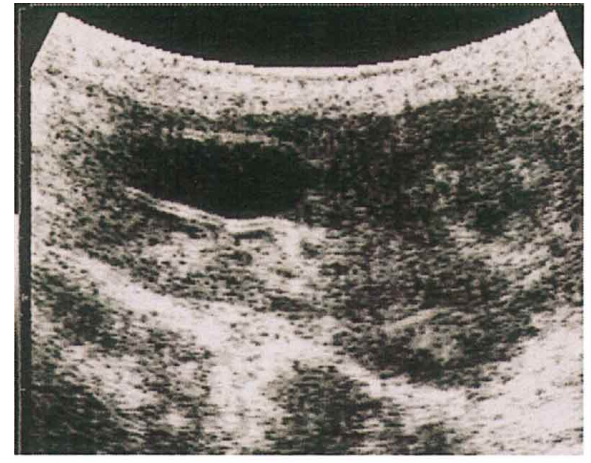

c)

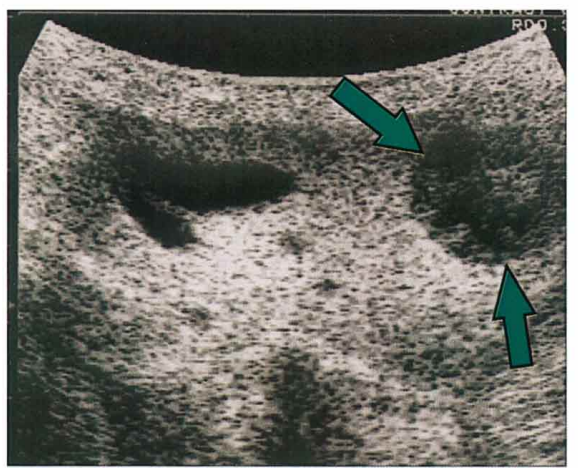

b)

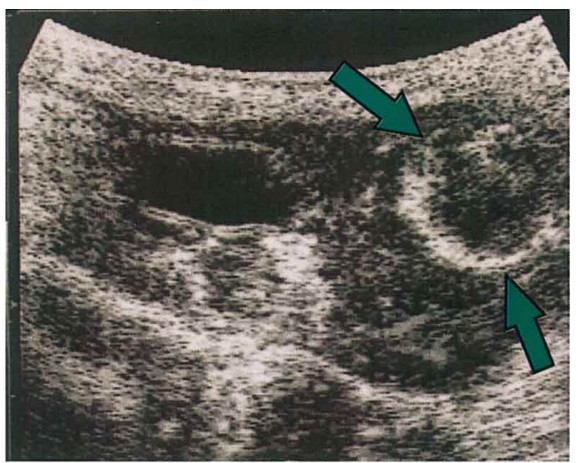

d)

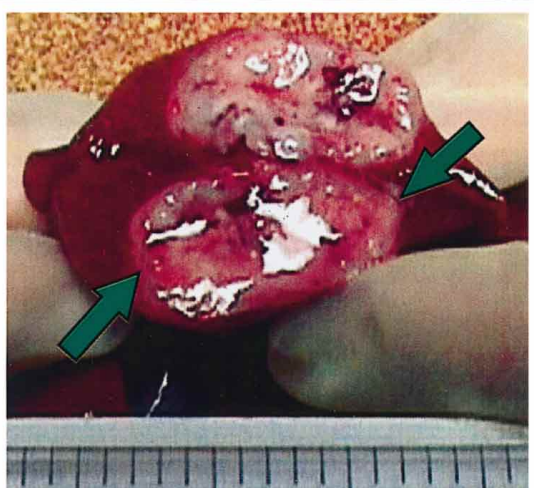

Fig. 4. The Liver of a VX-2 Tumor-Bearing Rabbit Imaged in the Harmonic Mode before (a), Immediately after (b), and 10 min after (c) Injection of $0.1 \mu 1$ Microbubbles/kg of Sonazoid 


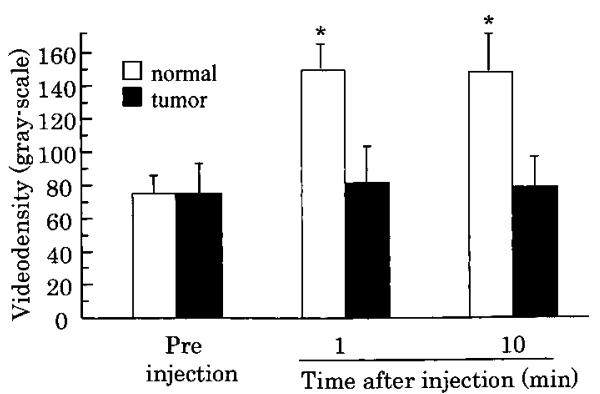

Fig. 5. Videodensitometric Measurements in the Normal Tissue (Open) and Tumor (Closed) of the Liver after Injection of Sonazoid in Rabbits $(0.1 \mu 1$ Microbubbles $/ \mathrm{kg}$, Conventional Mode)

Differences when $* p<0.05$ were compared with pre-injection values (Dunnett).

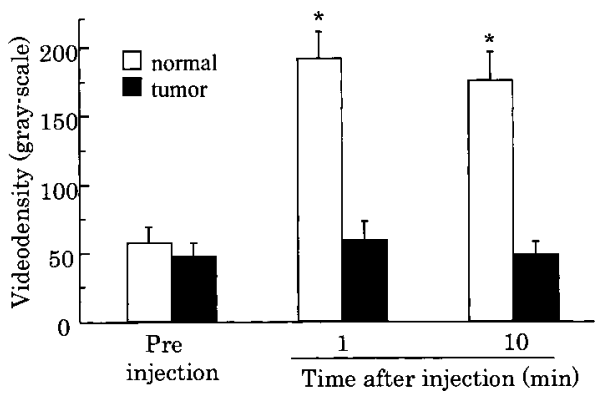

Fig. 6. Videodensitometric Measurements in the Normal Tissue (Open) and Tumor (Closed) of the Liver after Injection of Sonazoid in Rabbits $(0.1 \mu 1$ Microbubbles $/ \mathrm{kg}$, Harmonic Mode)

Differences when $* p<0.05$ were compared with pre-injection values (Dunnett)

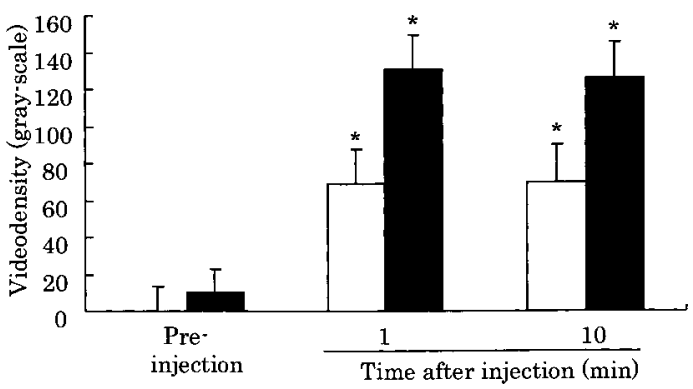

Fig. 7. Contrast of Videodensitometric Measurements between the Tumor and Normal Tissues of the Liver after Injection of Sonazoid $(0.1 \mu \mathrm{l} \mathrm{Mi}-$ crobubbles $/ \mathrm{kg}$ ) in Rabbits in Conventional (Open) and Harmonic (Closed) Modes

Differences of $* p<0.05$ were compared with pre-injection values (Dunnett).

the normal liver tissue and the tumor, especially in the case of such small tumors as this experiment. As the vessel-rich area at the tumor edge was not enhanced in the delayed phase, tumor depiction was improved. Additionally, homogeneous enhancement of the parenchyma in the delayed phase seemed to raise tumor conspicuity. Although visual scores of the early phase was not so good as those of the delayed phase in this study, the vascular imaging in the early phase had the advantage of allowing the identification of vessels and their differentiation from tumors or other lesions. The qualitative diagnosis of a focal lesion generally depends on the feature of vasculature, because malignant tumors develop neovascularity, and in the case of the liver, on the dominancy of the blood supply; poorly differentiated tumors have little or no
Table 1-1. Detection of the Liver Lesions before and after the Injection of Sonazoid $(0.1 \mu 1$ microbubbles $/ \mathrm{kg})$ in Rabbits

Conventional mode

\begin{tabular}{cccc}
\hline \hline Detection type & Before injection & Early phase & Delayed phase \\
\hline A & $0 / 18$ & $2 / 18$ & $7 / 18$ \\
B & $2 / 18$ & $5 / 18$ & $4 / 18$ \\
C & $9 / 18$ & $5 / 18$ & $2 / 18$ \\
D & $7 / 18$ & $6 / 18$ & $5 / 18$ \\
\end{tabular}

Harmonic mode

\begin{tabular}{cccc}
\hline \hline Detection type & Before injection & Early phase & Delayed phase \\
\hline $\mathrm{A}$ & $0 / 21$ & $7 / 21$ & $12 / 21$ \\
$\mathrm{~B}$ & $2 / 21$ & $6 / 21$ & $9 / 21$ \\
$\mathrm{C}$ & $4 / 21$ & $3 / 21$ & $0 / 21$ \\
$\mathrm{D}$ & $15 / 21$ & $5 / 21$ & $0 / 21$ \\
\hline
\end{tabular}

Table 1-2. Delineation of the Liver Lesions before and after the Injection of Sonazoid $(0.1 \mu 1$ microbubbles $/ \mathrm{kg})$ in Rabbits Conventional mode

\begin{tabular}{cccc}
\hline \hline Delineation score & Before injection & Early phase & Delayed phase \\
\hline 3 & $0 / 18$ & $1 / 18$ & $7 / 18$ \\
2 & $7 / 18$ & $12 / 18$ & $6 / 18$ \\
1 & $11 / 18$ & $5 / 18$ & $5 / 18$ \\
\hline
\end{tabular}

Harmonic mode

\begin{tabular}{cccc}
\hline \hline Delineation score & Before injection & Early phase & Delayed phase \\
\hline 3 & $1 / 21$ & $6 / 21$ & $12 / 21$ \\
2 & $5 / 21$ & $10 / 21$ & $9 / 21$ \\
1 & $15 / 21$ & $5 / 21$ & $0 / 21$
\end{tabular}

Each lesion was assessed before injection, during the early phase (injection to $1 \mathrm{~min}$ after injection) and the delayed phase (from $10 \mathrm{~min}$ after injection) by three independent observers. Assessments before injection were all performed in the conventional mode, and those after injection were performed in either the conventional (six rabbits) or harmonic (seven rabbits) mode. The liver lesions detected during imaging were subjectively classified according to the criteria described in 'Materials and Methods'.

blood supply of the portal vein, which is dominant in the healthy liver. ${ }^{1-3)}$ As the vascularity in, or around tumors becomes strongly enhanced, the early phase of Sonazoid-aided imaging will contribute well to the diagnosis of hepatic tumor malignancy.

In another imaging modality, MRI, RES-specific contrast in the liver parenchyma has been clinically available. Superparamagnetic iron oxide particles, ferumoxide, are taken up by Kupffer cells in the liver and thereby increase the contrast between normal tissue and tumors, which lack Kupffer cells. ${ }^{25)}$ This RES-specific contrast of ferumoxide in MRI is reported to show excellent specificity in hepatic tumor detection. If the microbubbles of Sonazoid would distribute in Kupffer cells as is postulated above, its usefulness in tumor detection similar to that demonstrated by ferumoxide would be expected. US is more convenient than MRI, because it can be done at the bedside of patients; and Sonazoid is more advantageous for repeated diagnosis than ferumoxide, because the contrast disappears almost completely on the next day with Sonazoid while contrast is prolonged for approximately 1 week with ferumoxide. ${ }^{26)}$

A former generation US agent, Levovist, which has also 
been described as having a liver-specific phase, ${ }^{27)}$ increases the conspicuity of liver metastases and renders occult lesions visible by using stimulated acoustic emission (SAE). ${ }^{28,29)}$ Levovist microbubbles are disrupted by high acoustic power and produce transient ultrasonic signals, an effect referred to as SAE. As sound pressures increase, the SAE signals intensify. They soon disappear, however, due to rapid destruction of the microbubbles. In this study, Sonazoid showed contrast effects sufficient for tumor detection with low acoustic power. A low-power setting will prolong the duration of the contrast effects and enable more detailed inspection. Thus, Sonazoid might be useful in searching for liver metastases after a diagnosis of tumors in other organs without any additional contrast agent injection.

In both studies, contrast enhancement in the harmonic mode tended to be better than that in the conventional mode. Harmonic mode is a novel US technology that allows visualization of non-linear backscatter signals (harmonics) from microbubbles in gray-scale: the ultrasound beam is transmitted at a fundamental frequency and the backscatter signal is received at harmonic frequency. ${ }^{30,31)}$ The efficacy of this technique is due to the difference in backscattering of the tissue and contrast agent at the two frequencies. Since the magnitude of the backscattered signal of the contrast agent at the harmonic frequency is greater than that of the tissue, the harmonic mode improves the signal-to-noise ratio and visualizes the distribution of contrast agent microbubbles more effectively. Therefore, the harmonic mode is more advantageous for contrast imaging than the conventional mode, ${ }^{32,33)}$ and some former generation contrast agents have diagnostic utility only in the harmonic imaging mode because they cannot show contrast effect on tissue perfusion in the conventional mode. ${ }^{9,34,35)}$ Although Sonazoid was also more efficient in the harmonic mode, it provided significant contrast enhancement in the VX-2 tumor model (Figs. 5-7). Thus this agent might be useful for tumor diagnosis by propagated and conventional ultrasonographic equipment.

In conclusion, Sonazoid rendered a contrast effect in the liver in two phases: early phase for vascular imaging and delayed phase for parenchymal imaging in both conventional and harmonic modes. In the rabbit VX-2 tumor model, the videodensitometric difference between tumor and normal tissues was comparable and significant, and visual evaluation scores displayed obvious improvement after injection in both modes. Sonazoid might be useful for the detection of undifferentiated tumors in the liver by visualization of neovascularity in the early phase and clear contrast defects in the delayed phase, not only in the harmonic but also in the conventional mode.

\section{REFERENCES}

1) Itai Y., Matsui O., Radiolog., 202, 306-314 (1997).

2) Matsui O., Kadoya M., Kameyama T., Yoshikawa J., Takashima T., Nakanuma Y., Unoura M., Kobayashi K., Izumi R., Ida M., Kitagawa K., Radiology, 209, 135-140 (1998).
3) Kim Ah. Y., Choi B. I., Kim T. K., Han J. K., Yun E. J., Lee K. Y., Han M. C., Radiology, 209, 135-140 (1998).

4) Tanaka S., Kitamura T., Fujita M., Nakanishi K., Okuda S., Am. J. Roentgenol., 154, 509—514 (1990).

5) Nico-Murcia M., Ralls P. W., Jeffrey R. B., Johnson M., Am. J. Roentgenol., 159, 1195-1197 (1992).

6) Lencioni R., Pinto F., Armillotta N., Bartolozzi C., Radiology, 201, 353-358 (1996).

7) Kudo M., Hepatogastroenterology, 45, 1226-1231 (1998).

8) Porter T. R., Xie F., Kricsfeld D., Armbruster P. W., Am. Coll. Cardiol., 27, 1497-1501 (1996).

9) Kono Y., Moriyasu F., Mine Y., Nada T., Kamiyama A., Suginoshita Y., Matsumura T., Kobayashi K., Chiba T., Invest. Radiol., 32, 120125 (1997).

10) Ding H., Kudo M., Onda H., Suetomi Y., Minami Y., Maekawa K., Radiology, 220, 349-356 (2001).

11) Hvattum E., Normann P. T., Oulie I., Steinar U., Rogstad O., Skotland T., J. Pharm. Biomed. Anal., 24, 487-494 (2001).

12) Kobayashi H., Beppu S., Hirayama H., Yagura S., Kashiwagi Y., Kayano H., J. Cardiol., 37, 209-214 (2001).

13) Forsberg F., Liu J-B., Merton D. A., Rawool N. M., Johnson D. K., Goldberg B. B., J. Ultrasound Med., 19, 557-563 (2000).

14) Tanaka M., Nakashima O., Wada Y., Kage M., Kojiro M., Hepatology, 24, 807-812 (1996).

15) Rous P., Beard J. W., J. Exp. Med., 62, 523-548 (1935).

16) Brown J. M., Quedens-Case C., Alderman J. L., Greener Y., Taylor K. J. W., Ultrasound Med. Biol., 24, 495-501 (1998).

17) Runge V. M., Invest. Radiol., 33, 263-267 (1998).

18) Schneider M., Broillet A., Bussat P., Giessinger N., Puginier J., Ventrone R., Yan F., Invest. Radiol., 32, 410-417 (1997).

19) Yao J., Teupe C., Takeuchi M., Avelar E., Sheahan M., Connolly R., Ostensen J., Pandian N. G., J. Am. Soc. Echocardiogr., 13, 570-581 (2000).

20) Hagen E. K., Forsberg F., Aksnes A., Merton D. A., Liu J., Tornes A., Johnson D., Goldberg B. B., Invest. Radiol., 35, 118-124 (2000).

21) Hagen E. K., Magnusson A., Aksnes A.-K., Norberg M., Acta Radiologica, 42, 225-229 (2001).

22) DeMaria A. N., Cotter B., Ohmori K., J. Am. Coll. Cardiol., 32, 1270-1271 (1998).

23) Dittrich H. C., Bales G. L., Kuvelas T., Hunt R. M., McFerran B. A., J. Am. Soc. Echocard., 8, 465-474 (1995).

24) Yamanouchi K., Tanaka M., Tsuda Y., Yokoyama K., Awazu S., Kobayashi Y., Chem. Pharm. Bull., 33, 1221-1231 (1985).

25) Oudkerk M., Van Den Heuvel A. G., Wielopolski P. A., Schmitz P. I. M., Borel Rinkes I. H. M., Wiggers T., Radiology, 203, 449-456 (1997).

26) Weissleder R., Radiology, 193, 593-595 (1994).

27) Heckemann R. A., Cosgrove D. O., Blomley M. J. K., Eckersley R. J., Harvey C. J., Mine Y., Radiology, 216, 592-596 (2000).

28) Blomley M. J. K., Albrecht T., Cosgrove D. O., Jayaram V., ButlerBarnes J., Eckersley R. J., Lancet, 351, 568 (1998).

29) Blomley M. J. K., Albrecht T., Cosgrove D. O., Patel N., Jayaram V., Butler-Barnes J., Eckersley R. J., Bauer A., Schlief R., Radiology, 210, 409-416 (1999).

30) Schrope B. A., Newhous V. L., Ultrasound Med. Biol., 19, 567-579 (1993).

31) Frinking P. J. A., Bouakaz A., Kirkhorn J., Cate F. J. T., de Jong N., Ultrasound Med. Biol., 26, 965-975 (2000).

32) Forsberg F., Goldberg B. B., Liu J., Merton D. A., Rawool N. M., J. Ultrasound Med., 15, 853-860 (1996).

33) Girard M. S., Mattrey R. F., Baker T., Peterson T., Deiranieh L. H., Steinbach G. C., J. Ultrasound Med., 19, 185-192 (2000).

34) Calliada F., Campani R., Bottinelli O., Bozzini A., Sommaruga M. G., Eur. J. Radiol., 27, S157-S160 (1998).

35) Geiser E. A., Cunningham D. J., Brown C. S., Wilson D. C., Ehocardiography, 17, 209-219 (2000). 